\title{
RECENT GINGA RESULTS ON GALACTIC X-RAY BINARIES
}

\author{
Y. Tanaka \\ Institute of Space and Astronautical science \\ 3-1-1 Yoshinodai, Sagamihara, Kanagawa-ken 229, Japan
}

\begin{abstract}
Based on the recent Ginga results, following topics on X-ray binaries are briefly discussed: The cyclotron resonnance features observed from several X-ray pulsars, and related problem of the magnetic field decay. Search for millisec. pulsations from LMXRBs. Very bright transients which are suspected to be new black hole candidates, and an estimation of the number of such black hole sources in our galaxy.
\end{abstract}

\section{Cyclotron features observed from X-ray pulsars}

The cyclotron resonnance features have been discoverd from at least three gamma-ray bursts (Murakami 1991), which made it convincing that the site of gamina-ray bursts is strongly magnetized neutron stars. Similar cyclotron features have also been found from nine accreting binary $X$-ray pulsars, which allow us to determine the magnetic fields of these neutron stars (Makishima 1990). The results are summarized in Table 1, and examples of the model fit to the observed spectruin are shown in Fig. 1. Thus derived field strengths are found to be confined in a fairly narrow range $(1-4) \times 10^{12}$ Gauss (see Table 1 ). In addition, the steep high-energy cut-off characteristics of the binary $X-$ ray pulsar spectra can now be understood as a result of the cyclotron resonnance scattering.

It is also possible to estimate the cyclotron energy, hence the magnetic field strength, from the spectral cut-off energy even if the cyclotron features are not clearly visible. For the above nine X-ray pulsars, the cyclotron energy is found to be no greater than twice the spectral cut-off energy (Makishima 1990). In fact, few binary X-ray pulsars show cut-off energies much less than $10 \mathrm{keV}$ or greater than 30 $\mathrm{keV}$. Thus, the observed concentration of the field strengths in a narrow range does not seem to be a selection effect.

It is important to note that at least three binary $X$-ray pulsars with high magnetic fields, Her X-1, X1626-67, and GX1+4, have low-mass companions. This fact and the evidence for high magnetic fields of the gamma-ray burst sources (neutron stars as old as $10^{9}$ yrs) do not support the magnetic field decay of neutron stars, independent of whether they 
are accreting or not, on a time scale shorter than $10^{8} \mathrm{yrs}$. The possibility of a different origin for weakly magnetized neutron stars in LMXRBs still remains open.

Table 1. Spectral parameters of binary X-ray pulsars

\begin{tabular}{llcccc}
\hline $\begin{array}{l}\text { Source } \\
\text { Name }\end{array}$ & Companion & $\begin{array}{c}\text { Orbital } \\
\text { Period(d) }\end{array}$ & $\begin{array}{c}\text { Pulse } \\
\text { Period(s) }\end{array}$ & $\begin{array}{c}\text { Spcctral } \\
\text { Ec (keV) }\end{array}$ & $\begin{array}{c}\text { Parameters } \\
\text { En (keV) }\end{array}$ \\
\hline Her X-1 & A9-B (HZ Her) & 1.7 & 1.24 & $17-21$ & 35 \\
4 U0115+63 & Be-transient & 24.3 & 3.6 & $7-9$ & $12,23,36$ \\
X0331+53 & $\begin{array}{c}\text { Be(BQ Cam)- } \\
\text { transient }\end{array}$ & 34.3 & 4.38 & $14-17$ & $28.0, \sim 53$ \\
Cep X-4 & Be?-transient & $?$ & 66.3 & $15-17$ & 32 \\
$4 U 1907+09$ & OB or Be & 8.4 & 438 & $14-16$ & 20 \\
$4 \mathrm{U} 1538-52$ & B0I (QV Nor) & 3.74 & 530 & $14-16$ & 20 \\
Vela X-1 & B0.5Ib (GP Vel) & 8.96 & 283 & $15-20$ & 27,53 \\
GX301-2 & B1.5Ia (BP Cru) & 41.5 & 690 & $19-21$ & 40 \\
X2259+58 & Single? & $?$ & 6.9 & & $\sim 7 ?, \sim 13 ?$ \\
\hline
\end{tabular}
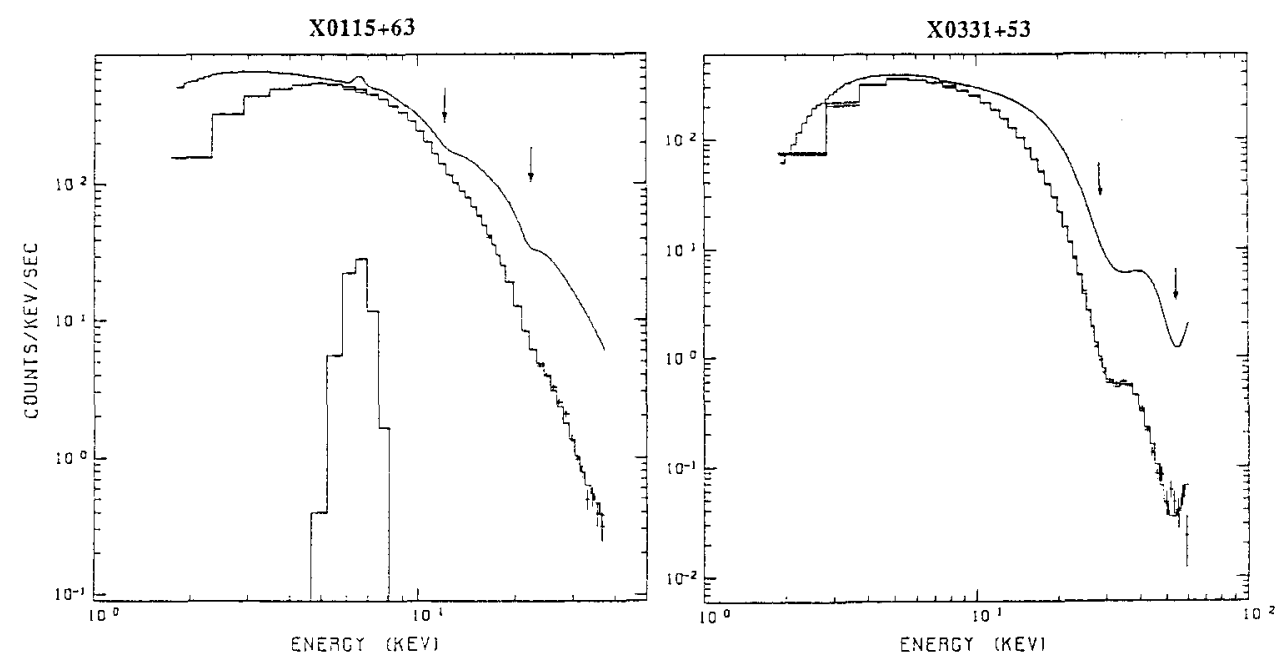

Fig. 1. Observed spectra of $X$-ray pulsars $X 0115+63$ and $X 0331+53$, fitted with the cyclotron model. Solid curves are the incident model spectra. 
2. Search for millisecond pulsations from LMXRBs

For the "recycled pulsar" scenario for millisec. radio pulsars, it is crucial that neutron stars in LMXRBs be millisec rotators. An extensive effort has been put to search for millisec. pulsations from several bright LMXRBs utilizing a large photon collecting power of Ginga. However, the results have so far been negative: the 95\% confidence upper limits determined for the amplitude of modulation are from $0.5 \%$ to $0.2 \%$ depending on the source intensity (Wood et al. 1990). After the fact, one can think of possible effects which smear out millisec. modulations in X-rays. Alternatively, more sensitive way may be to search for millisec radio pulsars from transient LMXRBs when they are X-ray quiescent. Such attempts are in progress.

\section{Very bright transients suspected to be black hole candidates}

Among several black hole candidates in our galaxy and LMC, Cyg $X-1$ and 10620-00 are considered to be the best two for which the mass lower limit of the compact objects is most likely greater than 3Mo (e.g. McClintock 1990). The X-ray characteristics of Cyg X-1 and A0620-00 are qualitatively different from each other, as sumarized in Table 2. Cyg $X-1$ has a single power law spectrum in its hard state and exhibits rapid chaotic variation (flickering). On the other hand, A0620-00 has an ultrasoft spectrum (much softer than LMXRBs) with a hard power-law tail, and shows little flickering. Because of the distinct nature of these characteristics from those of $X$-ray binaries which are known to include neutron stars, those sources which exhibit $X$-ray properties very similar to either of Cyg X-1 and A0620-00 have also been considered as the black hole candidates (see Table 2).

Table 2. X-ray properties of the black hole candidates

\begin{tabular}{lll}
\hline Spectrum & Time Variation & Sources \\
\hline $\begin{array}{l}\text { Hard-state } \\
\quad \text { Approximately }\end{array}$ & Flickering & Cyg X-1* \\
a single power-law & & GX339-4* \\
& & GS2023+33 \\
Ultrasoft-state & GS1826-24 \\
Ultrasoft & Little flickering & Cyg X-1* \\
& & GX339-4* \\
& & LMC X-3 \\
& & LMC X-1 \\
& & A0620-00 \\
& & GS2000+23 \\
& & GS1124-68*
\end{tabular}

* Occasionally switches between the hard- and ultrasoft-states. 
Ginga discovered three extremely bright transients, GS2000+25, GS2023 +33 , and GS1124-68, all of which are suspected to be black holes from their X-ray properties. GS2000+25 and GS1124-68 closely resemble A062000 , and GS2023+33 behaves very similar to Cyg X-1 in its hard state. (For the detail of these transients, see Tanaka 1989 \& 1991 and the references therein).

In addition, the light curves of these transients shown in Fig. 2 . together with that of A0620-00 look strikingly alike; a smooth exponential decay with a time constant of $\sim 40$ days, a second enhancement at about 60-70 days after outburst for at least three sources. Although what determines the light curve is not well understood yet, the similarity of the decay curve should reflect similarity of the physical properties among these binary systems.

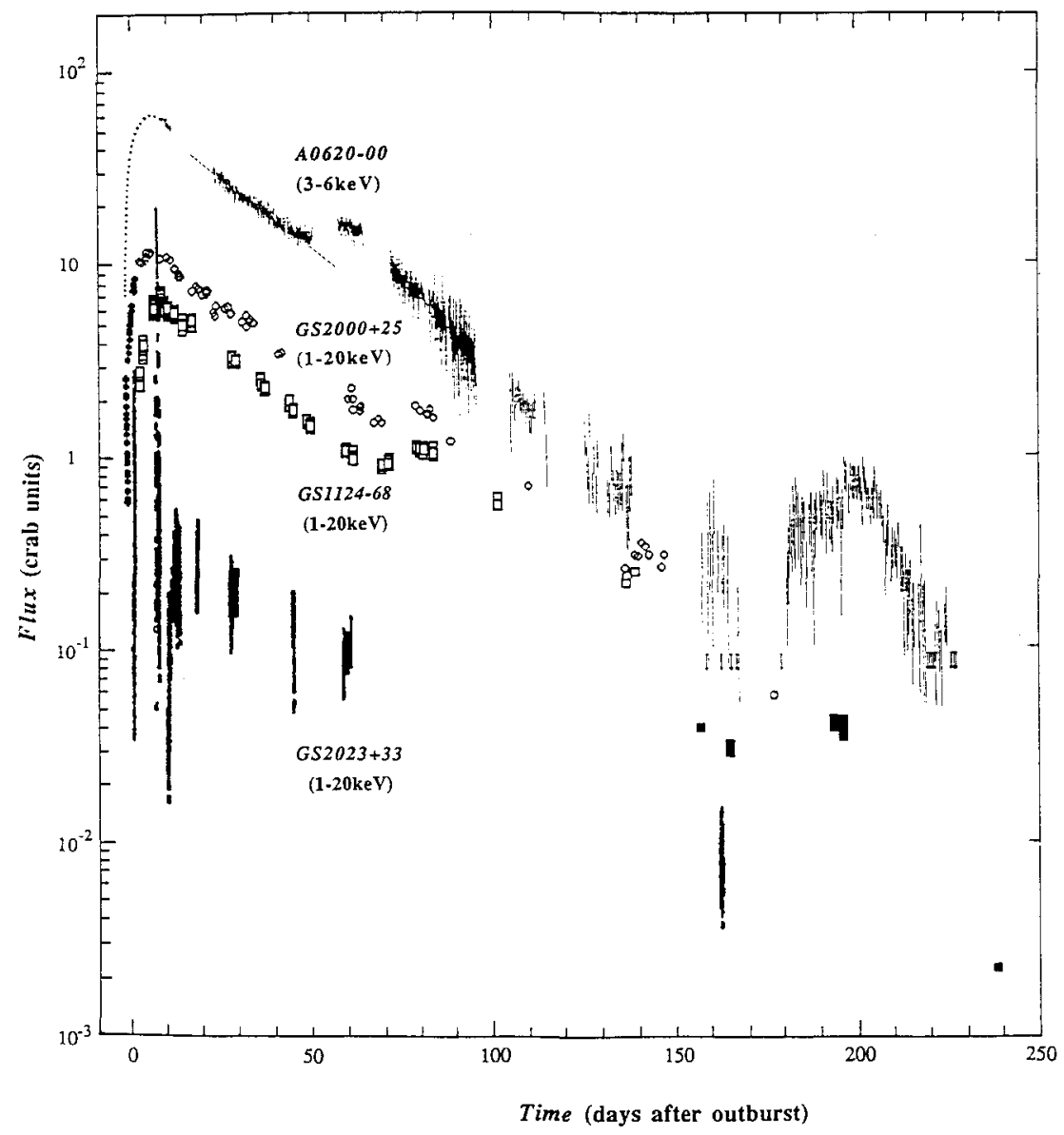

Fig. 2. The X-ray light curves of GS2000+25, GS2023+33, and GS1124-68. The X-ray light curve of A0620-00 is also shown for comparison. 
4. Number of transient black hole sources in our galaxy

There have been recorded as many as ten bright transients which are suspected to be black hole sources, including those detected by Ginga. At least six of them showed the energy spectrum composed of an ultrasoft component and a power-law component, and two of them a single power-law spectrum and exhibiting a pronounced flickering. The rate of detection of such transients has been roughly $0.5-1$ per year.

The efficiency of detection from the previous satellites and the recurrence rate of the individual transients are fairly uncertain. However, a crude estimate suggests that there exist at least a hundred and possibly as many as one thousand of such transient black hole sources in our galaxy. This number is comparable to or may even be greater than that of neutron star LMXRBs.

In addition, at least five of these transients were discovered associated with optical novae, and none identified with a massive star. This fact strongly suggests that the black hole transients belong to a low-mass binary systems. Since Cyg X-1, LMC X-1, and LMC X-3 (all of them are persistently luminous) are the only massive binary systems among known black hole candidates, majority of the black holes in our galaxy seems to reside in the low-mass systems. The origin of the black holes in low-mass binary systems has become an important new issue.

\section{REFERENCES.}

Makishima,K, (1990), Proc. U.S.-Japan Joint Seminar on the Structure and Evolution of Neutron Stars, 1990, Kyoto.

McClintock,J.E., (1990) Proc. Texas-ESO-CERN Symp. on Relativistic Astrophys., 1990. (The New York Academy of Sciences)

Murakami,T., (1991) Adv. Space Res. 11, (8)119.

Tanaka,Y., (1989) Proc. 23rd ESLAB Symp., 1989, Bologna, 1, p.3.

Tanaka,Y., (1991) Proc. Symposium on "X-Ray Binaries and the Formation of Binary and Millisecond Radio Pulsars", 1991, Santa Barbara.

Wood,K.S. et al., (1990) Astrophys. J. 379, 295. 